\title{
Growth and fixed points of solutions to second-order LDE with certain analytic coefficients in the unit disc
}

Jin Tu ${ }^{1 *}$, Ting-Yan Peng ${ }^{1}$, Hong-Yan X ${ }^{2}$, Hong Zhang ${ }^{3}$ and Guo-Yuan Dai ${ }^{1}$

"Correspondence:

tujin2008@sina.com

${ }^{1}$ College of Mathematics and

Information Science, Jiangxi Normal

University, Nanchang, 330022, China

Full list of author information is

available at the end of the article

\begin{abstract}
In this article, the authors investigate the growth and fixed points of solutions of certain second-order linear differential equations with analytic coefficients in the unit disc and obtain some results which improve and generalize previous results.

MSC: $30 \mathrm{D} 35 ; 34 \mathrm{M} 10$
\end{abstract}

Keywords: unit disc; hyper-order; fixed points

\section{Introduction and results}

In this paper, we shall assume that the readers are familiar with the fundamental results and standard notations of the Nevanlinna value distribution theory in the complex plane $\mathbb{C}$ and in the unit disc $\Delta=\{z:|z|<1\}$ (see [1-6]). Before we state our main results, we need to recall some definitions and notations.

Definition 1.1 $([1,2,5])$ For a meromorphic function $f(z)$ in $\Delta$, the order of $f(z)$ is defined by

$$
\sigma(f)=\varlimsup_{r \rightarrow 1^{-}} \frac{\log ^{+} T(r, f)}{\log \frac{1}{1-r}}
$$

where $T(r, f)$ is the characteristic function of $f(z)$. And for an analytic function $f(z)$ in $\Delta$, we define $\sigma_{M}(f)$ by

$$
\sigma_{M}(f)=\varlimsup_{r \rightarrow 1^{-}} \frac{\log ^{+} \log ^{+} M(r, f)}{\log \frac{1}{1-r}}
$$

where $M(r, f)=\max _{|z|=r}|f(z)|$ is the maximum modulus function of $f(z)$.

Remark $1.2([5])$ If $f(z)$ is an analytic function in $\Delta$, then

$$
\sigma(f) \leq \sigma_{M}(f) \leq \sigma(f)+1
$$

○2013 Tu et al.; licensee Springer. This is an Open Access article distributed under the terms of the Creative Commons Attribution License (http://creativecommons.org/licenses/by/2.0), which permits unrestricted use, distribution, and reproduction in any medium, provided the original work is properly cited. 
Definition $1.3([7,8])$ Let $f(z)$ be a meromorphic function in $\Delta$, the hyper-order of $f(z)$ is defined by

$$
\sigma_{2}(f)=\varlimsup_{r \rightarrow 1^{-}} \frac{\log ^{+} \log ^{+} T(r, f)}{\log \frac{1}{1-r}}=\varlimsup_{r \rightarrow 1^{-}} \frac{\log _{2}^{+} T(r, f)}{\log \frac{1}{1-r}} .
$$

If $f(z)$ is an analytic function in $\Delta$, then the hyper-order about maximum modulus of $f(z)$ is also defined by

$$
\sigma_{M, 2}(f)=\varlimsup_{r \rightarrow 1^{-}} \frac{\log _{3}^{+} M(r, f)}{\log \frac{1}{1-r}} .
$$

Definition 1.4 Let $f(z)$ be a meromorphic function in $\Delta$, the hyper-lower-order of $f(z)$ is defined by

$$
\mu_{2}(f)=\lim _{r \rightarrow 1^{-}} \frac{\log _{2}^{+} T(r, f)}{\log \frac{1}{1-r}} .
$$

If $f(z)$ is an analytic function in $\Delta$, we define $\mu_{M, 2}(f)$ by

$$
\mu_{M, 2}(f)=\lim _{r \rightarrow 1^{-}} \frac{\log _{3}^{+} M(r, f)}{\log \frac{1}{1-r}} .
$$

Remark 1.5 ([7]) If $f(z)$ is an analytic function in $\Delta$, then
(i) $\sigma_{2}(f)=\sigma_{M, 2}(f)$
(ii) $\mu_{2}(f)=\mu_{M, 2}(f)$.

Definition 1.6 The hyper convergence exponent and the hyper-lower convergence exponent of fixed points of a meromorphic function $f$ in $\Delta$ are defined by

$$
\lambda_{2}(f-z)=\varlimsup_{r \rightarrow 1^{-}} \frac{\log _{2}^{+} N\left(r, \frac{1}{f-z}\right)}{\log \frac{1}{1-r}}, \quad \underline{\lambda}_{2}(f-z)=\lim _{r \rightarrow 1^{-}} \frac{\log _{2}^{+} N\left(r, \frac{1}{f-z}\right)}{\log \frac{1}{1-r}} .
$$

And we also define $\bar{\lambda}_{2}(f-z)$ and $\bar{\lambda}_{2}(f-z)$, respectively, by

$$
\bar{\lambda}_{2}(f-z)=\varlimsup_{r \rightarrow 1^{-}} \frac{\log _{2}^{+} \bar{N}\left(r, \frac{1}{f-z}\right)}{\log \frac{1}{1-r}} \quad \text { and } \quad \bar{\lambda}_{2}(f-z)=\lim _{r \rightarrow 1^{-}} \frac{\log _{2}^{+} \bar{N}\left(r, \frac{1}{f-z}\right)}{\log \frac{1}{1-r}} \text {. }
$$

Many authors investigate the linear differential equation

$$
f^{\prime \prime}+A(z) e^{a z} f^{\prime}+B(z) e^{b z} f=0,
$$

where $A(z), B(z) \neq \equiv 0$ are entire functions (e.g., see [9-12]). In [10], Chen proved that if $a b \neq 0$ and $a \neq b$, then every solution $f(z) \neq 0$ of (1.1) is of infinite order; furthermore, if $a b \neq 0, a \neq b, A(z) \equiv 1, B(z)$ is a polynomial, then every solution $f(z) \not \equiv 0$ of (1.1) satisfies $\sigma_{2}(f)=1$. In 2012, Hamouda investigated the equation

$$
f^{\prime \prime}+A(z) e^{\frac{a}{\left(z_{0}-z\right)^{\mu}}} f^{\prime}+B(z) e^{\frac{b}{\left(z_{0}-z\right)^{\mu}}} f=0
$$

where $A(z)$ and $B(z)$ are analytic functions in $\Delta$, and he obtained the following results. 
Theorem 1.7 ([13]) Let $A(z)$ and $B(z) \not \equiv 0$ be analytic functions in the unit disc. Suppose that $\mu>1$ is a real constant, $a, b$ and $z_{0}$ are complex numbers such that $a b \neq 0, \arg a \neq \arg b$, $\left|z_{0}\right|=1$. If $A(z)$ and $B(z)$ are analytic on $z_{0}$, then every solution $f(z) \not \equiv 0$ of $(1.2)$ is of infinite order.

Theorem 1.8 ([13]) Let $A(z)$ and $B(z) \not \equiv 0$ be analytic functions in the unit disc. Suppose that $\mu>1$ is a real constant, $a, b$ and $z_{0}$ are complex numbers such that $a b \neq 0, a=c b$ $(0<c<1),\left|z_{0}\right|=1$. If $A(z)$ and $B(z)$ are analytic on $z_{0}$, then every solution $f(z) \neq \equiv$ of $(1.2)$ is of infinite order.

Remark 1.9 Throughout this paper, we choose the principal branch of logarithm of the function $e^{\frac{\lambda}{\left(z_{0}-z\right)^{\mu}}}$ if $\mu$ is not an integer $(\lambda \in \mathbb{C} \backslash 0)$.

In this paper, we focus on studying the hyper-order and fixed points of the solutions of (1.2) and obtain the following results.

Theorem 1.10 Let $A(z)$ and $B(z) \not \equiv 0$ be analytic functions in the unit disc, and let $\mu>1$ $b e$ a real constant, $a, b$ and $z_{0}$ be complex numbers such that $a b \neq 0, \arg a \neq \arg b,\left|z_{0}\right|=1$. If $A(z)$ and $B(z)$ satisfy one of the following conditions:

(1) $\max \left\{\sigma_{M}(A), \sigma_{M}(B)\right\} \leq \mu, A(z)$ and $B(z)$ are analytic on $z_{0}$;

(2) $\sigma_{M}(A)<\mu, \sigma_{M}(B) \leq \mu$ and $B(z)$ is analytic on $z_{0}$;

then every solution $f(z) \not \equiv 0$ of $(1.2)$ satisfies

(i) $\mu_{M, 2}(f)=\mu_{2}(f)=\sigma_{2}(f)=\sigma_{M, 2}(f)=\mu$;

(ii) $\bar{\lambda}_{2}(f-z)=\underline{\lambda}_{2}(f-z)=\bar{\lambda}_{2}(f-z)=\lambda_{2}(f-z)=\mu$.

Theorem 1.11 Under the assumptions of Theorem 1.10, with the exception that $a b \neq 0$, $a=c b(0<c<1),\left|z_{0}\right|=1$, every solution $f(z) \not \equiv 0$ of $(1.2)$ satisfies

(i) $\mu_{M, 2}(f)=\mu_{2}(f)=\sigma_{2}(f)=\sigma_{M, 2}(f)=\mu$;

(ii) $\underline{\lambda}_{2}(f-z)=\underline{\lambda}_{2}(f-z)=\bar{\lambda}_{2}(f-z)=\lambda_{2}(f-z)=\mu$.

Corollary 1.12 Let $A(z), B(z) \not \equiv 0, C(z)$ and $D(z)$ be analytic functions in the unit disc, and let $a, b$ and $z_{0}$ be complex numbers such that $a b \neq 0, \arg a \neq \arg b$ or $a=c b(0<c<1)$, $\left|z_{0}\right|=1$. If one of the following conditions holds,

(1) $\max \left\{\sigma_{M}(A), \sigma_{M}(B), \sigma_{M}(C), \sigma_{M}(D)\right\} \leq \mu$ and $A(z), B(z), C(z), D(z)$ are analytic on $z_{0}$;

(2) $\max \left\{\sigma_{M}(A), \sigma_{M}(C), \sigma_{M}(D)\right\}<\mu, \sigma_{M}(B) \leq \mu$ and $B(z)$ is analytic on $z_{0}$;

then every solution $f(z) \not \equiv 0$ of

$$
f^{\prime \prime}+\left(A(z) e^{\frac{a}{\left(z_{0}-z\right)^{\mu}}}+C(z)\right) f^{\prime}+\left(B(z) e^{\frac{b}{\left(z_{0}-z\right)^{\mu}}}+D(z)\right) f=0
$$

satisfies

(i) $\mu_{M, 2}(f)=\mu_{2}(f)=\sigma_{2}(f)=\sigma_{M, 2}(f)=\mu$;

(ii) $\bar{\lambda}_{2}(f-z)=\underline{\lambda}_{2}(f-z)=\bar{\lambda}_{2}(f-z)=\lambda_{2}(f-z)=\mu$.

Theorem 1.13 Let $A(z)$ and $B(z) \not \equiv 0$ be analytic functions in the unit disc. Suppose that $\mu>1$ and $v$ are real constants, $a, b, z_{0}$ and $z_{1}$ are complex numbers such that $b \neq 0, z_{0} \neq z_{1}$ and $\left|z_{0}\right|=\left|z_{1}\right|=1$. If $A(z)$ and $B(z)$ satisfy one of the following conditions:

(1) $A(z)$ and $B(z)$ are analytic on $z_{0}$;

(2) $\sigma_{M}(A)<\mu$ and $B(z)$ is analytic on $z_{0}$; 
then every solution $f(z) \not \equiv 0$ of

$$
f^{\prime \prime}+A(z) e^{\frac{a}{\left(z_{1}-z\right)^{\nu}}} f^{\prime}+B(z) e^{\frac{b}{\left(z_{0}-z\right)^{\mu}}} f=0
$$

satisfies $\mu_{M, 2}(f) \geq \mu_{2}(f) \geq \mu$.

Theorem 1.14 Let $A(z)$ and $B(z) \not \equiv 0$ be analytic functions in the unit disc. Suppose that $\mu, v(\mu>1, \mu \geq v)$ are real constants, $a, b, z_{0}$ and $z_{1}$ are complex numbers such that $b \neq 0$, $z_{0} \neq z_{1}$ and $\left|z_{0}\right|=\left|z_{1}\right|=1$. If $A(z)$ and $B(z)$ satisfy one of the following conditions:

(1) $\max \left\{\sigma_{M}(A), \sigma_{M}(B)\right\} \leq \mu, A(z)$ and $B(z)$ are analytic on $z_{0}$;

(2) $\sigma_{M}(A)<\mu, \sigma_{M}(B) \leq \mu$ and $B(z)$ is analytic on $z_{0}$;

then every solution $f(z) \not \equiv 0$ of $(1.4)$ satisfies

(i) $\mu_{2}(f)=\mu_{M, 2}(f)=\sigma_{2}(f)=\sigma_{M, 2}(f)=\mu$;

(ii) $\underline{\lambda}_{2}(f-z)=\underline{\lambda}_{2}(f-z)=\bar{\lambda}_{2}(f-z)=\lambda_{2}(f-z)=\mu$.

\section{Lemmas}

Lemma 2.1 ([14]) Let $k$ and $j$ be integers satisfying $k>j \geq 0$, and let $\varepsilon>0$. Iff $(z)$ is meromorphic in $\Delta$ such that $f^{(j)}$ does not vanish identically, then

$$
\left|\frac{f^{(k)}(z)}{f^{(j)}(z)}\right| \leq\left(\left(\frac{1}{1-|z|}\right)^{2+\varepsilon} \cdot \max \left\{\log \frac{1}{1-|z|}, T(s(|z|), f)\right\}\right)^{k-j} \quad\left(|z| \notin E_{1}\right)
$$

where $E_{1} \subset[0,1)$ is a set with $\int_{E_{1}} \frac{1}{1-r} d r<\infty$ and $s(|z|)=1-d(1-|z|)$.

Lemma $2.2([13])$ Let $A(z)$ be an analytic function on a point $z_{0} \in \mathbb{C}$, set $g(z)=A(z) e^{\frac{a}{\left(z-z_{0}\right)^{\mu}}}$ ( $\mu>0$ is a real constant), $a=\alpha+i \beta \neq 0, z_{0}-z=R e^{i \varphi}, \delta_{a}(\varphi)=\alpha \cos (\mu \varphi)+\beta \sin (\mu \varphi)$ and $H=\left\{\varphi \in[0,2 \pi): \delta_{a}(\varphi)=0\right\}$ (obviously, $H$ is of linear measure zero). Then, for any given $\varepsilon>0$ and for any $\varphi \in[0,2 \pi) \backslash H$, there exists $R_{0}>0$ such that for $0<R<R_{0}$, we have

(i) if $\delta_{a}(\varphi)>0$, then

$$
\exp \left\{(1-\varepsilon) \delta_{a}(\varphi) \frac{1}{R^{\mu}}\right\} \leq|g(z)| \leq \exp \left\{(1+\varepsilon) \delta_{a}(\varphi) \frac{1}{R^{\mu}}\right\}
$$

(ii) if $\delta_{a}(\varphi)<0$, then

$$
\exp \left\{(1+\varepsilon) \delta_{a}(\varphi) \frac{1}{R^{\mu}}\right\} \leq|g(z)| \leq \exp \left\{(1-\varepsilon) \delta_{a}(\varphi) \frac{1}{R^{\mu}}\right\}
$$

$\operatorname{Remark} 2.3([13])$ Set $\delta_{a}(\varphi)=\gamma \cos \left(\mu \varphi+\varphi_{0}\right)$, where $\gamma=\sqrt{\alpha^{2}+\beta^{2}}, \mu>1, \varphi \in[0,2 \pi)$. It is easy to know that $\delta_{a}(\varphi)$ changes its sign on each interval $I \subset[0,2 \pi)$ satisfying $\mu \cdot m I>\pi$, where $m I$ denotes the linear measure of the interval $I$.

Lemma $2.4([15])$ Let $g:(0,1) \rightarrow R$ and $h:(0,1) \rightarrow R$ be monotone increasing functions such that $g(r) \leq h(r)$ holds outside of an exceptional set $E_{2} \subset[0,1)$, for which $\int_{E_{2}} \frac{1}{1-r} d r<\infty$. Then there exists a constant $d \in(0,1)$ such that if $s(r)=1-d(1-r)$, then $g(r) \leq h(s(r))$ for all $r \in[0,1)$. 
Lemma 2.5 ([7]) If $A_{0}(z), A_{1}(z), \ldots, A_{k-1}(z)$ are analytic functions of finite order in the unit disc, then every solution $f \not \equiv 0$ of

$$
f^{(k)}+A_{k-1} f^{(k-1)}+\cdots+A_{1} f^{\prime}+A_{0} f=0
$$

satisfies

$$
\sigma_{2}(f)=\sigma_{M, 2}(f) \leq \max \left\{\sigma_{M}\left(A_{j}\right): j=0,1, \ldots, k-1\right\} .
$$

Remark 2.6 Lemma 2.5 is a special case of Theorem 2.1 in [7].

Lemma 2.7 ([4]) Let $f$ be a meromorphic function in the unit disc, and let $k \in \mathbb{N}$. Then

$$
m\left(r, \frac{f^{(k)}}{f}\right)=S(r, f)
$$

where $S(r, f)=O\left(\log ^{+} T(r, f)\right)+O\left(\log \left(\frac{1}{1-r}\right)\right)$, possibly outside a set $E_{3} \subset[0,1)$ with $\int_{E_{3}} \frac{1}{1-r} d r<\infty$.

Lemma 2.8 ([8]) Suppose that $A_{0}, A_{1}, \ldots, A_{k-1}, F \not \equiv 0$ are meromorphic functions in $\Delta$, and let $f(z)$ be a meromorphic solution of the equation

$$
f^{(k)}+A_{k-1} f^{(k-1)}+\cdots+A_{0} f=F(z),
$$

such that $\max \left\{\sigma_{i}(F), \sigma_{i}\left(A_{j}\right): j=0,1, \ldots, k-1\right\}<\sigma_{i}(f)$, where $i=1,2$, then

$$
\bar{\lambda}_{i}(f)=\lambda_{i}(f)=\sigma_{i}(f) .
$$

Lemma 2.9 Suppose that $A_{0}, A_{1}, \ldots, A_{k-1}, F \not \equiv 0$ are meromorphic functions in $\Delta$, and let $f(z)$ be a meromorphic solution of equation (2.2) such that $\max \left\{\sigma_{i}(F), \sigma_{i}\left(A_{j}\right): j=0,1, \ldots, k-\right.$ $1\}<\mu_{i}(f)$, where $i=1,2$, then

$$
\underline{\underline{\lambda}}_{i}(f)=\underline{\lambda}_{i}(f)=\mu_{i}(f) .
$$

Proof Suppose that $f(z) \not \equiv 0$ is a solution of (2.2), by (2.2), we get

$$
\frac{1}{f}=\frac{1}{F}\left(\frac{f^{(k)}}{f}+A_{k-1} \frac{f^{(k-1)}}{f}+\cdots+A_{0}\right),
$$

it is easy to see that if $f$ has a zero at $z_{0}$ of order $\alpha(\alpha>k)$, and $A_{0}, \ldots, A_{k-1}$ are analytic at $z_{0}$, then $F$ must have a zero at $z_{0}$ of order $\alpha-k$, hence

$$
n\left(r, \frac{1}{f}\right) \leq k \bar{n}\left(r, \frac{1}{f}\right)+n\left(r, \frac{1}{F}\right)
$$

and

$$
N\left(r, \frac{1}{f}\right) \leq k \bar{N}\left(r, \frac{1}{f}\right)+N\left(r, \frac{1}{F}\right) .
$$


By Lemma 2.7 and (2.3), we have

$$
m\left(r, \frac{1}{f}\right) \leq m\left(r, \frac{1}{F}\right)+\sum_{j=0}^{k-1} m\left(r, A_{j}\right)+O\left\{\log ^{+} T(r, f)+\log \left(\frac{1}{1-r}\right)\right\} \quad\left(r \notin E_{3}\right)
$$

where $\int_{E_{3}} \frac{1}{1-r} d r<\infty$. By (2.4)-(2.5), we get

$$
\begin{aligned}
T\left(r, \frac{1}{f}\right) \leq & k \bar{N}\left(r, \frac{1}{f}\right)+T(r, F)+\sum_{j=0}^{k-1} T\left(r, A_{j}\right) \\
& +O\left\{\log ^{+} T(r, f)+\log \left(\frac{1}{1-r}\right)\right\} \quad\left(r \notin E_{3}\right) .
\end{aligned}
$$

Since $\max \left\{\sigma_{i}(F), \sigma_{i}\left(A_{j}\right): j=0,1, \ldots, k-1\right\}<\mu_{i}(f)$, then we have

$$
\max \left\{\frac{T(r, F)}{T(r, f)}, \frac{T\left(r, A_{j}\right)}{T(r, f)}\right\} \rightarrow 0 \quad\left(r \rightarrow 1^{-}, j=0, \ldots, k-1\right)
$$

By (2.6)-(2.7) and by Lemma 2.4, for all $|z|=r \in[0,1)$, we have

$$
(1-o(1)) T(r, f) \leq k \bar{N}\left(s(r), \frac{1}{f}\right)+O\left\{\log ^{+} T(s(r), f)+\log \left(\frac{1}{1-s(r)}\right)\right\}
$$

where $s(r)=1-d(1-r), d \in(0,1)$. By $(2.8)$, we have

$$
\underline{\bar{\lambda}}_{i}(f)=\underline{\lambda}_{i}(f)=\mu_{i}(f) \quad(i=1,2)
$$

\section{Proofs of theorems}

Proof of Theorem 1.10 (i) Suppose that $f(z) \not \equiv 0$ is a solution of (1.2), we obtain

$$
\left|B(z) e^{\frac{b}{\left(z_{0}-z\right)^{\mu}}}\right| \leq\left|\frac{f^{\prime \prime}(z)}{f(z)}\right|+\left|A(z) e^{\frac{a}{\left(z_{0}-z\right)^{\mu}}}\right|\left|\frac{f^{\prime}(z)}{f(z)}\right|
$$

From Lemma 2.1, for any given $\varepsilon>0$, there exists a set $E_{1} \subset[0,1)$ with $\int_{E_{1}} \frac{1}{1-r} d r<\infty$ such that for all $z \in \Delta$ satisfying $|z|=r \notin E_{1}$, we have

$$
\left|\frac{f^{(k)}(z)}{f(z)}\right| \leq\left(\frac{1}{1-r}\right)^{M}[T(s(r), f)]^{k} \quad(k=1,2)
$$

where $s(r)=1-d(1-r), d \in(0,1), M>0$ is a constant, not necessarily the same at each occurrence. Set $I=\left\{\theta: z-z_{0}=R e^{i \theta}, z \in \Delta\right\} \subset[0,2 \pi)$, we have $m I \rightarrow \pi$ as $R \rightarrow 0$. Since $\mu>$ 1 , there exists $R_{0}$ ( $R_{0}$ is sufficiently small and not necessarily the same at each occurrence) such that for $0<R<R_{0}$, we have $\mu \cdot m I>\pi$. By Remark 2.3 and $\arg a \neq \arg b$, for all $0<$ $R<R_{0}$, there exists some $\varphi \in I$ such that $\delta_{b}(\varphi)>0$ and $\delta_{a}(\varphi)<0$. From Lemma 2.2, for any given $\varepsilon(0<\varepsilon<1)$ and for all $z \in\left\{z: z-z_{0}=R e^{i \varphi}, z \in \Delta\right\}$, there exists $R_{0}$ such that for $0<R<R_{0}$, we have

$$
\left|B(z) e^{\frac{b}{\left(z_{0}-z\right)^{\mu}}}\right| \geq \exp \left\{(1-\varepsilon) \delta_{b}(\varphi) \frac{1}{R^{\mu}}\right\} .
$$


By condition (1) that $A(z)$ is analytic on $z_{0}$ and by Lemma 2.2, for all $z \in E_{4}=\left\{z: z-z_{0}=\right.$ $\left.R e^{i \varphi}, 0<R<R_{0}, z \in \Delta\right\}$, we have

$$
\left|A(z) e^{\frac{a}{\left.z_{0}-z\right)^{\mu}}}\right| \leq \exp \left\{(1-\varepsilon) \delta_{a}(\varphi) \frac{1}{R^{\mu}}\right\}<M .
$$

By the metric relations in the triangle $\triangle o z_{0} z$, we have

$$
|z|^{2}=1+R^{2}-2 R \cos \varphi^{*} \quad\left(\varphi^{*}=\angle o z_{0} z \in\left[0, \frac{\pi}{2}\right)\right) .
$$

By (3.5), for all $z \in\left\{z: z-z_{0}=R e^{i \theta}, 0<R<R_{0}, \theta \in I\right\}$, there exists certain $\varepsilon_{0}\left(0<\varepsilon_{0}<1\right)$ such that

$$
\frac{1}{1-|z|} \geq \frac{1}{R}=\frac{1}{1-|z|} \frac{2 \cos \varphi^{*}-R}{1+|z|}>\frac{\varepsilon_{0}}{1-|z|} .
$$

Equation (3.6) implies $R \rightarrow 0 \Longleftrightarrow r \rightarrow 1^{-}$. By condition (2), $\sigma_{M}(A)<\mu$ and $\delta_{a}(\varphi)<0$, for all $z \in \Delta,|z|=r \rightarrow 1^{-}$and $0<R<R_{0}$, we have

$$
\left|A(z) e^{\frac{a}{\left(z_{0}-z\right)^{\mu}}}\right|=|A(z)| \exp \left\{\frac{\delta_{a}(\varphi)}{R^{\mu}}\right\} \leq M \exp \left\{\frac{1}{(1-r)^{\mu_{0}}}\right\},
$$

where $\mu_{0}$ satisfies $\sigma_{M}(A)<\mu_{0}<\mu$. By (3.1)-(3.4), (3.6) and (3.7), for all $z \in E_{4}$ and $|z|=r \notin$ $E_{1} \rightarrow 1^{-}$, we have

$$
\exp \left\{(1-\varepsilon) \delta_{b}(\varphi) \frac{\varepsilon_{0}{ }^{\mu}}{(1-r)^{\mu}}\right\} \leq M\left(\frac{1}{1-r}\right)^{M} \exp \left\{\frac{1}{(1-r)^{\mu_{0}}}\right\} \cdot[T(s(r), f)]^{2}
$$

where $s(r)=1-d(1-r), d \in(0,1)$. By (3.8) and Lemma 2.4, we have

$$
\mu \leq \mu_{2}(f)=\mu_{M, 2}(f) .
$$

On the other hand, by Lemma 2.5 , we have

$$
\sigma_{2}(f)=\sigma_{M, 2}(f) \leq \mu \text {. }
$$

By (3.9) and (3.10), we have

$$
\mu_{M, 2}(f)=\mu_{2}(f)=\sigma_{2}(f)=\sigma_{M, 2}(f)=\mu .
$$

(ii) Set $g(z)=f(z)-z, z \in \Delta$, where $f(z) \not \equiv 0$ is a solution of (1.2). It is obvious that $\bar{\lambda}_{2}(g)=$ $\bar{\lambda}_{2}(f-z), \lambda_{2}(g)=\lambda_{2}(f-z), \sigma_{2}(g)=\sigma_{2}(f-z)=\sigma_{2}(f)=\mu$. Then equation (1.2) becomes

$$
g^{\prime \prime}+A(z) e^{\frac{a}{\left(z_{0}-z\right)^{\mu}}} g^{\prime}+B(z) e^{\frac{b}{\left(z_{0}-z\right)^{\mu}}} g=-\left(A(z) e^{\frac{a}{\left(z_{0}-z\right)^{\mu}}}+z B(z) e^{\frac{b}{\left(z_{0}-z\right)^{\mu}}}\right) .
$$

By (3.3), (3.4) and (3.7), it is easy to see $A(z) e^{\frac{a}{\left.z_{0}-z\right)^{\mu}}}+z B(z) e^{\frac{b}{\left.z_{0}-z\right)^{\mu}}} \not \equiv 0$ by modulus estimation. By Lemma 2.8 and (3.11), we have

$$
\bar{\lambda}_{2}(g)=\lambda_{2}(g)=\sigma_{2}(g)=\sigma_{2}(f)=\mu \text {. }
$$


Also, by Lemma 2.9 and (3.11), we deduce $\underline{\bar{\lambda}}_{2}(g)=\underline{\lambda}_{2}(g)=\mu_{2}(g)=\mu$. Therefore, we obtain

$$
\underline{\bar{\lambda}}_{2}(f-z)=\underline{\lambda}_{2}(f-z)=\bar{\lambda}_{2}(f-z)=\lambda_{2}(f-z)=\mu \text {. }
$$

Proof of Theorem 1.11 (i) Similar to the proof of Theorem 1.10, we can obtain (3.1)-(3.3). Since $a=c b(0<c<1)$ and $\delta_{b}(\varphi)>0$, we have $\delta_{a}(\varphi)=c \delta_{b}(\varphi)>0$. From conditions (1)-(2) and by (3.7), Lemma 2.2, for any given $\varepsilon>0$ and for all $z \in E_{4}=\left\{z: z-z_{0}=R e^{i \varphi}, 0<R<\right.$ $\left.R_{0}, z \in \Delta\right\}$, we have

$$
\left|A(z) e^{\frac{a}{\left(z_{0}-z\right)^{\mu}}}\right| \leq \exp \left\{(1+\varepsilon) \delta_{a}(\varphi) \frac{1}{R^{\mu}}\right\}
$$

By (3.1)-(3.3) and (3.12), for any given $\varepsilon\left(0<\varepsilon<\frac{1-c}{1+c}\right)$ and for all $z \in E_{4}=\left\{z: z-z_{0}=R e^{i \varphi}, 0<\right.$ $\left.R<R_{0}, z \in \Delta\right\}$, we have

$$
\exp \left\{[(1-c-(1+c) \varepsilon)] \delta_{b}(\varphi) \frac{1}{R^{u}}\right\} \leq M\left(\frac{1}{1-r}\right)^{M} \cdot[T(s(r), f)]^{2} \quad\left(r \notin E_{1}\right)
$$

where $s(r)=1-d(1-r), d \in(0,1)$ and $\int_{E_{1}} \frac{1}{1-r} d r<\infty$. By (3.6) and Lemma 2.4, we obtain

$$
\exp \left\{[(1-c-(1+c) \varepsilon)] \delta_{b}(\varphi) \frac{\varepsilon_{0}^{\mu}}{(1-r)^{\mu}}\right\} \leq\left(\frac{1}{1-s(r)}\right)^{M} \cdot\left[T\left(s_{1}(r), f\right)\right]^{2} \quad\left(r \rightarrow 1^{-}\right)
$$

where $s_{1}(r)=1-d^{2}(1-r), d \in(0,1)$. By (3.13) and Lemma 2.4, we have

$$
\mu \leq \mu_{M, 2}(f)=\mu_{2}(f) .
$$

On the other hand, by Lemma 2.5, we have $\sigma_{2}(f)=\sigma_{M, 2}(f) \leq \mu$. Therefore, we obtain

$$
\mu_{M, 2}(f)=\mu_{2}(f)=\sigma_{2}(f)=\sigma_{M, 2}(f)=\mu \text {. }
$$

(ii) By the similar proof in case (ii) of Theorem 1.10, we have that

$$
\underline{\lambda}_{2}(f-z)=\underline{\lambda}_{2}(f-z)=\bar{\lambda}_{2}(f-z)=\lambda_{2}(f-z)=\mu
$$

holds for every solution $f(z) \not \equiv 0$ of (1.2).

Proof of Theorem 1.13 Suppose that $f \not \equiv 0$ is a solution of (1.4), from (1.4), we obtain

$$
\left|B(z) e^{\frac{b}{\left(z_{0}-z\right)^{\mu}}}\right| \leq\left|\frac{f^{\prime \prime}(z)}{f(z)}\right|+\left|A(z) e^{\frac{a}{\left(z_{1}-z\right)^{v}}}\right|\left|\frac{f^{\prime}(z)}{f(z)}\right|
$$

Since $A(z)$ is analytic on $z_{0}$ or $\sigma_{M}(A)<\mu_{0}<\mu$, for $z$ near enough $z_{0}$ and $z \in \Delta$, we have

$$
|A(z)|<M, \quad \text { or } \quad|A(z)|<\exp \left\{\frac{1}{(1-r)^{\mu_{0}}}\right\} \text {. }
$$

Since $z_{1} \neq z_{0}$, for all $z$ near enough $z_{0}$ and $z \in \Delta$, we have

$$
\left|z_{1}-z\right| \geq\left|z_{1}-z_{0}\right|-\left|z_{0}-z\right| \geq \frac{\left|z_{0}-z_{1}\right|}{2}, \quad\left|e^{\frac{a}{\left(z_{1}-z\right)^{v}}}\right| \leq e^{\frac{2^{v}|a|}{\left|z_{1}-z_{0}\right|^{v}}} \leq M
$$


Using (3.1)-(3.3), (3.6) and (3.15)-(3.16), for all $z \in E_{4}$ and $|z|=r \notin E_{1} \rightarrow 1^{-}$, we obtain

$$
\exp \left\{(1-\varepsilon) \delta_{b}(\varphi) \frac{\varepsilon_{0}^{\mu}}{(1-r)^{\mu}}\right\} \leq M\left(\frac{1}{1-r}\right)^{M} \exp \left\{\frac{1}{(1-r)^{\mu_{0}}}\right\} \cdot[T(s(r), f)]^{2}
$$

By (3.17) and Lemma 2.4, we have

$$
\mu \leq \mu_{2}(f)=\mu_{M, 2}(f) .
$$

Proof of Theorem 1.14 (i) From Theorem 1.13 we have that every solution $f(z) \not \equiv 0$ of (1.4) satisfies

$$
\mu \leq \mu_{2}(f)=\mu_{M, 2}(f) .
$$

On the other hand, by Lemma 2.5 , we have that every solution $f(z) \not \equiv 0$ of (1.4) satisfies

$$
\sigma_{2}(f)=\sigma_{M, 2}(f) \leq \mu \text {. }
$$

Therefore every solution $f(z) \not \equiv 0$ of (1.4) satisfies

$$
\mu_{M, 2}(f)=\mu_{2}(f)=\sigma_{2}(f)=\sigma_{M, 2}(f)=\mu .
$$

(ii) Set $g(z)=f(z)-z, z \in \Delta$, equation (1.4) becomes

$$
g^{\prime \prime}+A(z) e^{\frac{a}{\left(z_{1}-z\right)^{v}}} g^{\prime}+B(z) e^{\frac{b}{\left(z_{0}-z\right)^{\mu}}} g=-\left(A(z) e^{\frac{a}{\left(z_{1}-z\right)^{\nu}}}+z B(z) e^{\frac{b}{\left(z_{0}-z\right)^{\mu}}}\right) .
$$

It is easy to see $A(z) e^{\frac{a}{\left(z_{1}-z\right)^{\mu}}}+z B(z) e^{\frac{b}{\left(z_{0}-z\right)^{\mu}}} \not \equiv 0$ by (3.3), (3.15) and (3.16). By the similar proof in case (ii) of Theorem 1.10, we have that every solution $f(z) \not \equiv 0$ of (1.4) satisfies

$$
\underline{\lambda}_{2}(f-z)=\underline{\lambda}_{2}(f-z)=\bar{\lambda}_{2}(f-z)=\lambda_{2}(f-z)=\mu \text {. }
$$

\section{Competing interests}

The authors declare that they have no competing interests.

\section{Authors' contributions}

JT, TYP, HYX, HZ and GYD completed the main part of this article, JT corrected the main theorems. All authors read and approved the final manuscript.

\section{Author details}

${ }^{1}$ College of Mathematics and Information Science, Jiangxi Normal University, Nanchang, 330022, China. ${ }^{2}$ Department of Informatics and Engineering, Jing dezhen Ceramic Institute, Jingdezhen, Jiangxi 333403, China. ${ }^{3}$ School of the Tourism and Urban Management, Jiangxi University of Finance and Economics, Nanchang, 330013, China.

\section{Acknowledgements}

The authors thank the referees for their valuable suggestions to improve the present article. This project is supported by the National Natural Science Foundation of China (Grant No. 11171119, 11261024, 11271045, 11301233), the Natural Science Foundation of Jiangxi Province in China (Grant 20122BAB211005, 20132BAB211001, 20132BAB211002). 


\section{References}

1. Hayman, WK: Meromorphic Functions. Clarendon Press, Oxford (1964)

2. Heittokangas, J: On complex differential equations in the unit disc. Ann. Acad. Sci. Fenn., Math. Diss. 122, 1-54 (2000)

3. Laine, I: Nevanlinna Theory and Complex Differential Equations. de Gruyter, Berlin (1993)

4. Nevanlinna, R: Analytic Functions. Springer, New York (1970)

5. Tsuji, M: Potential Theory in Modern Function Theory, Reprinting of the 1959 edition. Chelsea, New York (1975)

6. Yang, L: Value Distribution Theory. Spring, Berlin (1993)

7. Cao, TB, Yi, HX: The growth of solutions of linear differential equations with coefficients of iterated order in the unit disc. J. Math. Anal. Appl. 319, 278-294 (2006)

8. Cao, TB: The growth oscillation and fixed points of solutions of complex linear differential equations in the unit disc J. Math. Anal. Appl. 352(2), 739-748 (2009)

9. Amemiya, I, Ozawa, M: Non-existence of finite order solutions of $w^{\prime \prime}+e^{-z} w^{\prime}+Q(z) w=0$. Hokkaido Math. J. 10, 1-17 (1981)

10. Chen, ZX: The growth of solutions of $f^{\prime \prime}+A(z) e^{z} f^{\prime}+Q(z) f=0$ where the order of $Q=1$. Sci. China Ser. A 45, 290-300 (2002)

11. Chen, ZX, Shon, $\mathrm{KH}$ : The relation between solutions of a class of second order differential equation with functions of small growth. Chin. Ann. Math., Ser. A 27(4), 431-442 (2006) (in Chinese)

12. Gundersen, GG: On the question of whether $f^{\prime \prime}+e^{-z} f^{\prime}+B(z) f=0$ can admit a solution $f \not \equiv 0$ of finite order. Proc. R. Soc. Edinb. A 102, 9-17 (1986)

13. Hamouda, S: Properties of solutions to linear differential equations with analytic coefficients in the unit disc. Electron. J. Differ. Equ. 177, 1-8 (2012)

14. Chyzhykov, I, Gundersen, GG, Heittokangas, J: Linear differential equations and logarithmic derivative estimates. Proc. Lond. Math. Soc. 86, 735-754 (2003)

15. Bank, S: General theorem concerning the growth of solutions of first order algebraic differential equations. Compos. Math. 25, 61-70 (1972)

10.1186/1687-1847-2013-342

Cite this article as: Tu et al.: Growth and fixed points of solutions to second-order LDE with certain analytic

coefficients in the unit disc. Advances in Difference Equations 2013, 2013:342

\section{Submit your manuscript to a SpringerOpen ${ }^{\ominus}$ journal and benefit from:}

- Convenient online submission

- Rigorous peer review

- Immediate publication on acceptance

- Open access: articles freely available online

- High visibility within the field

- Retaining the copyright to your article 